\title{
Multiple Solutions for the Discrete $p$-Laplacian Boundary Value Problems
}

\author{
Yuhua Long ${ }^{1,2}$ and Haiping $\mathrm{Shi}^{3}$ \\ ${ }^{1}$ School of Mathematics and Information Science, Guangzhou University, Guangzhou 510006, China \\ ${ }^{2}$ Key Laboratory of Mathematics and Interdisciplinary Sciences of Guangdong Higher Education Institute, Guangzhou University, \\ Guangzhou 510006, China \\ ${ }^{3}$ Modern Business and Management Department, Guangdong Construction Vocational Technology Institute, \\ Guangzhou 510450, China
}

Correspondence should be addressed to Haiping Shi; shp7971@163.com

Received 27 January 2014; Accepted 11 March 2014; Published 8 April 2014

Academic Editor: Elena Braverman

Copyright (C) 2014 Y. Long and H. Shi. This is an open access article distributed under the Creative Commons Attribution License, which permits unrestricted use, distribution, and reproduction in any medium, provided the original work is properly cited.

By employing a critical point theorem, established by Bonanno, we prove the existence of three distinct solutions to boundary value problems of nonlinear difference equations with a discrete $p$-Laplacian operator. To demonstrate the applicability of our results, we also present an example.

\section{Introduction}

In the present paper we will deal with the following discrete boundary value problem, with homogeneous Dirichlet conditions:

$$
\begin{gathered}
-\Delta \phi_{p}(\Delta u(t-1))+\phi_{p}(u(t))=\lambda f(t, u(t)) \\
t \in[1, T] \\
u(0)=u(T+1)=0,
\end{gathered}
$$

where $T \geq 2$ is an integer and $[1, T]$ denotes the discrete interval $\{1,2, \ldots, T\} . p>1$ is a real number; $\phi_{p}(s)=|s|^{p-2} s$ for all $s \in \mathbf{R}$. Here $\Delta$ is the forward difference operator defined by $\Delta u(t)=u(t+1)-u(t)$ for all $t \in \mathbf{Z}$, while $f:[1, T] \times \mathbf{R} \rightarrow \mathbf{R}$ is a continuous function. $\lambda$ is a nonnegative parameter.

Boundary value problems for difference equations have been extensively studied; see the monographs [1-5]. Difference equations represent the discrete counterpart of ordinary equations, and the classical theory of difference equations employs numerical analysis and features from the linear and nonlinear operator theory, such as fixed point methods; for an exhaustive description of the subject, we refer the reader to the monographs of Agarwal [1], Kelley and Peterson [6], and Lakshmikantham and Trigiante [3]. We remark that, usually, most methods yield existence results for solutions of a difference equation.

The issue of multiplicity of solutions can be investigated through variational methods. Recently, many results have been established by applying variational methods. For example, we may recall here the works of Cabada et al. $[7,8]$, Cai and Yu [9], Guo and Yu [10], and Deng et al. [11]. In all the aforementioned papers, the variational methods are applied to difference equations on discrete intervals and the variational approach represents an important advance as it allows proving multiplicity results as well.

In [7], the authors consider (1) in this form

$$
\begin{gathered}
-\Delta \phi_{p}(\Delta u(t-1))=\lambda f(t, u(t)) \quad t \in[1, T] \\
u(0)=u(T+1)=0
\end{gathered}
$$

and give conclusion that there exists $\lambda>0$ such that (2) admits at least three solutions under the following assumptions:

(F1) lim $\sup _{|t| \rightarrow+\infty}\left(F(k, t) /|t|^{p}\right) \leq 0$ for every $k \epsilon$ $[1, T]$;

(F2) $\sum_{k=1}^{T} \sup _{|t| \leq s / c_{1}} F(k, t)<\sum_{k=1}^{T} \sup _{t \in \mathbf{R}} F(k, t)$;

(F3) $\sup _{r / c_{2} \leq|t| \leq r / c_{1}} F(k, t) \leq-\sum_{h \neq k} \sup _{|t| \leq r / c_{1}} F(h, t)$ for every $k \in[1, T]$. 
In this paper, under convenient assumptions on the reaction term $f$, we not only give the result that (1) admits at least three distinct solutions but also find the two open intervals which $\lambda$ lies in and make estimation of the norm of $u$.

The rest of the paper has the following structure. In Section 2 we introduce the variational framework for problem (1) and transfer the existence of solutions of boundary value problem (1) into the existence of critical points of the corresponding functional. Employing the critical point theorem of Bonanno [12], we state our main results and give proofs of the main results in Section 3. Finally, we exhibit a simple example to demonstrate the applicability of our results.

\section{Variational Framework}

This section is devoted to the formulation of a variational framework for (1). We are going to define a suitable Banach space $(E,\|\cdot\|)$ and an energy functional $I \in \mathbf{C}^{1}(E)$, such that critical points of $I$ in $E$ are exact solutions of (1).

We define the real vector space

$$
E=\{u:[0, T+1] \longrightarrow \mathbf{R} \mid u(0)=u(T+1)=0\}
$$

and, for every $u \in E$, we denote

$$
\|u\|=\left(\sum_{t=1}^{T+1}|\Delta u(t-1)|^{p}+|u(t)|^{p}\right)^{1 / p} .
$$

So $(E,\|\cdot\|)$ is a reflexive Banach space and $\operatorname{dim}(E)=T$. We also put, for every $u \in E$,

$$
\|u\|_{\infty}=\max _{t \in[1, T]}|u(t)| .
$$

By classical results, the norms $\|\cdot\|$ and $\|\cdot\|_{\infty}$ are equivalent on $E$; then there exists a constant $c>0$ such that

$$
\|u\|_{\infty} \leq c\|u\| .
$$

For later use, here we give the estimation of constant $c$.

Lemma 1. The constant $c$ defined in (6) can be denoted as $c=$ $(T+1)^{1 / q}$, where $(1 / p)+(1 / q)=1$.
Proof. For every $u \in E$, given a $\tau \in[1, T+1]$, by Dirichlet conditions $u(0)=u(T+1)=0$ and Hölder inequality, we have

$$
\begin{aligned}
|u(t)|= & \left|u(\tau-1)+\sum_{s=\tau}^{t} \Delta u(s-1)\right| \\
\leq & |u(\tau-1)|+\sum_{s=1}^{T+1}|\Delta u(s-1)| \\
\leq & \sum_{s=1}^{T+1}|u(s-1)|+\sum_{s=1}^{T+1}|\Delta u(s-1)| \\
\leq & \left(\sum_{s=1}^{T+1} 1^{q}\right)^{1 / q}\left(\sum_{s=1}^{T+1}|u(s-1)|^{p}\right)^{1 / p} \\
& +\left(\sum_{s=1}^{T+1} 1^{q}\right)^{1 / q}\left(\sum_{s=1}^{T+1}|\Delta u(s-1)|^{p}\right)^{1 / p} \\
= & (T+1)^{1 / q}\left(\sum_{s=1}^{T+1}|u(s)|^{p}\right)^{1 / p} \\
& +(T+1)^{1 / q}\left(\sum_{s=1}^{T+1}|\Delta u(s-1)|^{p}\right)^{1 / p} \\
= & (T+1)^{1 / q}\|u\| .
\end{aligned}
$$

Define $\Phi: E \rightarrow \mathbf{R}$ and $J: E \rightarrow \mathbf{R}$ by

$$
\Phi(u)=\frac{1}{p}\|u\|^{p}, \quad J(u)=\sum_{t=1}^{T} F(t, u(t)),
$$

where $F(t, u)=\int_{0}^{u} f(t, s) d s$. Define $I: E \rightarrow \mathbf{R}$ by

$$
I(u)=\Phi(u)-\lambda J(u) .
$$

Lemma 2. For any $\lambda>0, I(u)$ is continuously Gâteaux differentiable, and, for every $u, v \in E$,

$$
\begin{gathered}
\left\langle I^{\prime}(u), v\right\rangle=\sum_{t=1}^{T}\left[\left(-\Delta \phi_{p}(\Delta u(t-1))+\phi_{p}(u(t))\right.\right. \\
-\lambda f(t, u(t))) \cdot v(t)] .
\end{gathered}
$$

Proof. Clearly $I \in \mathbf{C}^{1}(E, \mathbf{R})$; in what follows we prove (10). Choose $u, v \in E$; let $\varphi: \mathbf{R} \rightarrow \mathbf{R}$ be an arbitrary mapping; we recall the summation by parts formula

$$
\begin{aligned}
\sum_{t=1}^{T}[\varphi(\Delta u(t-1)) \Delta v(t-1)+\Delta \varphi(\Delta u(t-1)) v(t)] \\
=\varphi(\Delta u(T)) v(T) .
\end{aligned}
$$


Using (11) with $\varphi=\phi_{p}$ and $u(0)=u(T+1)=0$, we get

$$
\sum_{t=1}^{T+1} \phi_{p}(\Delta u(t-1)) \Delta v(t-1)=-\sum_{t=1}^{T} \Delta \phi_{p}(\Delta u(t-1)) v(t) .
$$

Then

$$
\begin{aligned}
& \lim _{\delta \rightarrow 0^{+}} \frac{\|u+\delta v\|^{p}-\|u\|^{p}}{p \delta} \\
&=\lim _{\delta \rightarrow 0^{+}}\left(\sum _ { t = 1 } ^ { T + 1 } \left(\left(|\Delta(u+\delta v)(t-1)|^{p}-|\Delta u(t-1)|^{p}\right)\right.\right. \\
&\left.\left.\quad+\left(|(u+\delta v)(t)|^{p}-|u(t)|^{p}\right)\right) \times(\delta p)^{-1}\right) \\
&=\sum_{t=1}^{T+1}\left(\phi_{p}(\Delta u(t-1)) \Delta v(t-1)+\phi_{p}(u(t)) v(t)\right) \\
&=\sum_{t=1}^{T}\left(-\Delta \phi_{p}(\Delta u(t-1)) v(t)+\phi_{p}(u(t)) v(t)\right) \\
&+\phi_{p}(u(T+1)) v(T+1) \\
&=\sum_{t=1}^{T}\left(-\Delta \phi_{p}(\Delta u(t-1))+\phi_{p}(u(t)) v(t)\right) .
\end{aligned}
$$

Besides, we have

$$
\begin{aligned}
\lim _{\delta \rightarrow 0^{+}} & \frac{J(u+\delta v)-J(u)}{\delta} \\
= & \lim _{\delta \rightarrow 0^{+}} \frac{\sum_{t=1}^{T} F(t,(u+\delta v)(t))-\sum_{t=1}^{T} F(t, u(t))}{\delta} \\
& =\sum_{t=1}^{T} \lim _{\delta \rightarrow 0^{+}} \frac{F(t,(u+\delta v)(t))-F(t, u(t))}{\delta} \\
& =\sum_{t=1}^{T} f(t, u(t)) v(t) .
\end{aligned}
$$

Equalities (13) and (14) imply (10).

From Lemma 2, we easily get the following lemma which yields a variational formulation for (1).

Lemma 3. For all $\lambda>0$, every critical point $u \in E$ of $I(u)$ is a solution of (1).

Proof. Fix $\lambda>0$ and assume that $u \in E$ is a critical point of $I$; then

$$
I^{\prime}(u)=0 .
$$

From Lemma 2, this is equivalent to

$\sum_{t=1}^{T}\left[\left(-\Delta \phi_{p}(\Delta u(t-1))+\phi_{p}(u(t))-\lambda f(t, u(t))\right) \cdot v(t)\right]=0$,

$\forall v \in E$
For any $h \in[0, T+1]$, we define $e_{h} \in E$ by putting $e_{h}(t)=\delta_{h t}$ for all $t \in[0, T+1]$, where $\delta_{h t}=1$ if $h=t$ and $\delta_{h t}=0$ if $h \neq t$. If we apply (16) with $v=e_{h}$, we have

$$
-\Delta \phi_{p}(\Delta u(t-1))+\phi_{p}(u(t))-\lambda f(t, u(t))=0
$$

this is exactly (1). So, $u$ solves (1); that is, $u$ is in fact a solution of (1).

\section{Main Results}

In order to present our main results, we introduce some notations at first. Denote $M=\max _{(t, u) \in[1, T] \times[-\alpha, \alpha]} F(t, u)$; then write

$$
\varphi_{1}=\frac{c^{p} p T M}{\alpha^{p}}, \quad \varphi_{2}=\frac{p\left(\sum_{t=1}^{T} F(t, \beta)-T M\right)}{T \beta^{p}},
$$

and, for each $h>1$,

$$
b=\frac{h T(\alpha \beta)^{p}}{p \alpha^{p} \sum_{t=1}^{T} F(t, \beta)-p T^{2}(c \beta)^{p} M} .
$$

Now, we state the following convenient assumptions on the function $f$.

$\left(H_{1}\right)$ There exist two constants $\alpha>0, \beta>0$ with $\alpha<c \beta T^{1 / p}$ such that

$$
M<\frac{\alpha^{p}}{T\left(\alpha^{p}+T(c \beta)^{p}\right)} \sum_{t=1}^{T} F(t, \beta),
$$

where $c$ is defined in (6).

$\left(H_{2}\right)$ There exist constants $a_{1}>0, a_{2}>0, R>0$, and $0<\mu<p$ such that

$$
F(t, u) \leq a_{1}|u|^{\mu}+a_{2}, \quad \forall|u| \geq R .
$$

Remark 4. If $\left(H_{1}\right)$ holds, then

$$
b>0 .
$$

In fact, from $\left(H_{1}\right)$, we have

$$
T M<\frac{\alpha^{p}}{\alpha^{p}+T(c \beta)^{p}} \sum_{t=1}^{T} F(t, \beta) ;
$$

it follows that

$$
\begin{aligned}
T^{2} p(c \beta)^{p} M & <\frac{\alpha^{p} T p(c \beta)^{p}}{\alpha^{p}+T(c \beta)^{p}} \sum_{t=1}^{T} F(t, \beta) \\
& =\frac{\alpha^{p} p}{\left(\alpha^{p} / T(c \beta)^{p}\right)+1} \sum_{t=1}^{T} F(t, \beta)<\alpha^{p} p \sum_{t=1}^{T} F(t, \beta) ;
\end{aligned}
$$

therefore,

$$
\alpha^{p} p \sum_{t=1}^{T} F(t, \beta)-T^{2} p(c \beta)^{p} M>0
$$


which means that

$$
b>0 .
$$

With the above preparations, we present our main results.

Theorem 5. Assume that $\left(H_{1}\right)$ and $\left(H_{2}\right)$ hold. Then, for each

$$
\lambda \in \Lambda_{1}=\left(\frac{1}{\varphi_{2}}, \frac{1}{\varphi_{1}}\right) .
$$

Equation (1) admits at least three solutions in $E$ and, moreover, there exist an open interval $\Lambda_{2} \subseteq[0, b]$ and a positive real number $\sigma$ such that, for each $\lambda \in \Lambda_{2}$, (1) admits at least three solutions in $E$ whose norms in $E$ are less than $\sigma$.

We replace $\left(\mathrm{H}_{2}\right)$ by the following.

$\left(H_{2}^{\prime}\right)$ There exist constants $R>0$ and $0<\mu<p$ such that

$$
0<u f(t, u) \leq \mu F(t, u), \quad \forall|u| \geq R .
$$

We have the following.

Corollary 6. If $\left(H_{1}\right)$ and $\left(H_{2}^{\prime}\right)$ hold, conclusions in Theorem 5 are true.

Remark 7. Integrate (28); it follows (21). Then conclusions in Corollary 6 are true.

When $f(t, u)$ is independent of $t$, consider the following special case of (1):

$$
\begin{gathered}
-\Delta \phi_{p}(\Delta u(t-1))+\phi_{p}(u(t))=\lambda f(u(t)) \\
t \in[1, T] \\
u(0)=u(T+1)=0,
\end{gathered}
$$

where $f \in \mathbf{C}(\mathbf{R}, \mathbf{R})$.

Denote $\widetilde{M}=\max _{u \in[-\alpha, \alpha]} F(u)$; we define

$$
\widetilde{\varphi}_{1}=\frac{c^{p} p T \widetilde{M}}{\alpha^{p}}, \quad \widetilde{\varphi}_{2}=\frac{p(F(\beta)-\widetilde{M})}{\beta^{p}},
$$

and, for each $h>1$,

$$
\widetilde{b}=\frac{h(\alpha \beta)^{p}}{p \alpha^{p} F(\beta)-p T(c \beta)^{p} \widetilde{M}} .
$$

We need the following assumptions.

$\left(\widetilde{H_{1}}\right)$ There exist two constants $\alpha>0, \beta>0$ with $\alpha<c \beta T^{1 / p}$ such that

$$
\widetilde{M}<\frac{\alpha^{p}}{\alpha^{p}+T(c \beta)^{p}} F(\beta) .
$$

$\left(\widetilde{H_{2}}\right)$ There exist constants $\widetilde{a_{1}}>0, \widetilde{a_{2}}>0, R>0$, and $0<\mu<p$ such that

$$
F(u) \leq \widetilde{a_{1}}|u|^{\mu}+\widetilde{a_{2}}, \quad \forall|u| \geq R .
$$

Remark 8. If $\left(\widetilde{H_{1}}\right)$ holds, then $\widetilde{b}>0$.

Similarly, we obtain the following.

Theorem 9. Assume that $\left(\widetilde{H_{1}}\right)$ and $\left(\widetilde{H_{2}}\right)$ hold. Then, for each

$$
\lambda \in \Lambda_{3}=\left(\frac{1}{\widetilde{\varphi}_{2}}, \frac{1}{\widetilde{\varphi}_{1}}\right) .
$$

Equation (29) admits at least three solutions in $E$ and, moreover, there exist an open interval $\Lambda_{4} \subseteq[0, \widetilde{b}]$ and $a$ positive real number $\sigma$ such that, for each $\lambda \in \Lambda_{4}$, (29) admits at least three solutions in $E$ whose norms in $E$ are less than $\sigma$.

Corollary 10. Let $f(u)$ satisfy $\left(\widetilde{H_{1}}\right)$ and $\left(\widetilde{H_{2}^{\prime}}\right)$ there exist constants $R>0$ and $0<\mu<p$ such that

$$
0<u f(u) \leq \mu F(u), \quad \forall|u| \geq R .
$$

Then the conclusions in Theorem 9 also hold.

To give proofs of our results, we need the following three critical point theorems, established by Bonanno [12].

For the reader's convenience, we recall the definition of weak closure.

Suppose that $E \subset X$. We denote $\bar{E}^{\omega}$ as the weak closure of $E$, that is, $x \in \bar{E}^{\omega}$, if there exists a sequence $\left\{x_{n}\right\} \subset E$ such that $f\left(x_{n}\right) \rightarrow f(x)$ for every $f \in X^{*}$.

Lemma 11 (see [12]). Let $X$ be a separable and reflexive real Banach space; let $\Phi: X \rightarrow \mathbf{R}$ be a nonnegative continuously Gâteaux differentiable and sequentially weakly lower semicontinuous functional whose Gâteaux derivative admits a continuous inverse on $X^{*}$; let $J: X \rightarrow \mathbf{R}$ be a continuously Gâteaux differentiable functional whose Gâteaux derivative is compact. Assume that there exists $x_{0} \in X$ such that $\Phi\left(x_{0}\right)=J\left(x_{0}\right)=0$ and that

(i) $\lim _{\|x\| \rightarrow+\infty}(\Phi(x)-\lambda J(x))=+\infty$ for all $\lambda \geq 0$;

(ii) there are $r>0$ and $x_{1} \in X$ such that $r<\Phi\left(x_{1}\right)$;

(iii) $\sup _{\overline{x \in \Phi^{-1}((-\infty, r))}} \omega(x)<\left(r /\left(r+\Phi\left(x_{1}\right)\right)\right) J\left(x_{1}\right)$.

Then for each

$$
\begin{gathered}
\lambda \in \Lambda=\left(\frac{\Phi\left(x_{1}\right)}{J\left(x_{1}\right)-\sup _{\overline{x \in \Phi^{-1}((-\infty, r))}} \omega(x)},\right. \\
\left.\frac{r}{\sup _{\overline{x \in \Phi^{-1}((-\infty, r))}} \omega(x)}\right)
\end{gathered}
$$

the equation

$$
\Phi^{\prime}(x)-\lambda J^{\prime}(x)=0
$$

has at least three solutions in $X$, and, for each $h>1$, there exist an open interval

$$
\widetilde{\Lambda} \subseteq\left[0, \frac{h r}{\left(r J\left(x_{1}\right) / \Phi\left(x_{1}\right)\right)-\sup _{\overline{x \in \Phi^{-1}((-\infty, r))}} \omega(x)}\right)
$$

and a positive real number $\sigma$ such that, for each $\lambda \in \widetilde{\Lambda}$, (37) has at least three solutions in $X$ whose norms are less than $\sigma$. 
With the aid of Lemma 11, we devote ourselves to giving proof of Theorem 5 .

Proof of Theorem 5. Let space $X$ be the Banach space E. It follows from (8) that $\Phi$ is a nonnegative Gâteaux differentiable and weakly lower semicontinuous functional, whose Gâteaux derivative admits a continuous inverse on $X^{*}$, and let $J: X \rightarrow \mathbf{R}$ be a continuously Gâteaux differentiable functional whose Gâteaux derivative is compact. Moreover, $\Phi(0)=J(0)=0$.

In view of $\left(H_{2}\right)$, we have, for any $u \in E,|u| \geq R$ for each $t \in[0, T+1]$ and $\lambda \geq 0$,

$$
\begin{aligned}
\Phi(u)-\lambda J(u) & =\frac{1}{p}\|u\|^{p}-\lambda \sum_{t=1}^{T} F(t, u(t)) \\
& \geq \frac{1}{p}\|u\|^{p}-\lambda \sum_{t=1}^{T}\left(a_{1}|u|^{\mu}+a_{2}\right) \\
& \geq \frac{1}{p}\|u\|^{p}-\lambda a_{1} T\|u\|_{\infty}^{\mu}-\lambda a_{2} T \\
& \geq \frac{1}{p}\|u\|^{p}-\lambda a_{1} T c^{\mu}\|u\|^{\mu}-\lambda a_{2} T .
\end{aligned}
$$

Since $0<\mu<p$, it follows that $\lim _{\|x\| \rightarrow+\infty}(\Phi(x)-\lambda J(x))=$ $+\infty$ for all $\lambda \geq 0$. Condition (i) of Lemma 11 is satisfied.

Now, we put

$$
r=\frac{\alpha^{p}}{c^{p} p}, \quad u_{1}(t)= \begin{cases}0, & t=0 \\ \beta, & t \in[1, T] \\ 0, & t=T+1 .\end{cases}
$$

It is clear that $u_{1}(t) \in E$ and

$$
\begin{aligned}
\Phi\left(u_{1}\right)= & \frac{1}{p}\left\|u_{1}\right\|^{p}=\sum_{t=1}^{T+1}\left(\left|\Delta u_{1}(t-1)\right|^{p}+\left|u_{1}(t)\right|^{p}\right) \\
= & \sum_{t=1}^{T}\left|u_{1}(t)\right|^{p}=\frac{1}{p} T \beta^{p} \\
& J\left(u_{1}\right)=\sum_{t=1}^{T} F\left(t, u_{1}(t)\right)=\sum_{t=1}^{T} F(t, \beta) .
\end{aligned}
$$

In view of $\alpha<c \beta T^{1 / p}$, together with (41), it yields

$$
\Phi\left(u_{1}\right)=\frac{\left\|u_{1}\right\|^{p}}{p}=\frac{T \beta^{p} c^{p}}{p c^{p}}>\frac{\alpha^{p}}{c^{p} p}=r
$$

which implies that the assumption (ii) of Lemma 11 holds.

Finally, we verify that the assumption (iii) of Lemma 11 is true to complete the proof of Theorem 5 .

Note that the estimate $\Phi(u) \leq r$ implies that

$$
|u|^{p} \leq c^{p}\|u\|^{p} \leq c^{p} p \Phi(u) \leq c^{p} p r
$$

for all $t \in[0, T+1]$. By the definition of $r$ (see (40)) and (44), we obtain

$$
|u|^{p} \leq \alpha^{p}, \quad \forall t \in[0, T+1] .
$$

This means that

$$
\Phi^{-1}((-\infty, r)) \subseteq\{u \in E|| u(t) \mid \leq \alpha, t \in[0, T+1]\} .
$$

Thus, for all $u \in E$, we have

$$
\frac{\sup }{u \in \Phi^{-1}((-\infty, r))} \omega_{\omega} J(u)=\sup _{u \in \Phi^{-1}((-\infty, r))} J(u) \leq T M .
$$

On the other hand, we get

$$
\begin{aligned}
\frac{r}{r+\Phi\left(u_{1}\right)} J\left(u_{1}\right) & =\frac{r p}{r p+T \beta^{p}} \sum_{t=1}^{T} F(t, \beta) \\
& =\frac{\alpha^{p}}{\alpha^{p}+T(c \beta)^{p}} \sum_{t=1}^{T} F(t, \beta)
\end{aligned}
$$

which shows that (iii) of Lemma 11 is satisfied.

Note that

$$
\begin{gathered}
\frac{\Phi\left(u_{1}\right)}{J\left(u_{1}\right)-\sup _{u \in \Phi^{-1}((-\infty, r))}^{\omega} J(u)} \leq \frac{T \beta^{p}}{p\left(\sum_{t=1}^{T} F(t, \beta)-T M\right)}, \\
\frac{r}{\sup _{u \in \Phi^{-1}((-\infty, r))} \omega(u)} \geq \frac{\alpha^{p}}{c^{p} p T M} .
\end{gathered}
$$

By $\left(H_{1}\right)$, we obtain

$$
T M<\frac{\alpha^{p}}{\alpha^{p}+T(c \beta)^{p}} \sum_{t=1}^{T} F(t, \beta)
$$

which implies

$$
\frac{c^{p} p T M}{\alpha^{p}}<\frac{p\left(\sum_{t=1}^{T} F(t, \beta)-T M\right)}{T \beta^{p}}
$$

Making use of this, together with (18) and (19), it follows that $\varphi_{2}>\varphi_{1}$. Combining (49) and (50) and applying Lemma 11, we achieve that, for each $\lambda \in \Lambda_{1}=\left(1 / \varphi_{2}, 1 / \varphi_{1}\right) \subset \Lambda$, (1) admits at least three solutions in $E$.

For each $h>1$, we note that

$$
\begin{aligned}
& \frac{h r}{r\left(J\left(u_{1}\right) / \Phi\left(u_{1}\right)\right)-\sup _{u \in \Phi^{-1}((-\infty, r))}} \omega(u) \\
& \quad \leq \frac{h T \alpha \beta^{p}}{p \alpha^{p} \sum_{t=1}^{T} F(t, \beta)-p T^{2}(c \beta)^{p} M}=b>0 .
\end{aligned}
$$

Then, from Lemma 11, it yields that, for each $h>1$, there exist an open interval $\Lambda_{2} \subseteq[0, b]$ and a real number $\sigma>0$, such that, for $\lambda \in \Lambda_{2}$, (1) admits at least three solutions in $E$ whose norms in $E$ are less than $\sigma$. And the proof of Theorem 5 is completed.

Similar to the proof of Theorem 5, we can give proof of Theorem 9; here we omit it.

Finally, we exhibit a simple example to demonstrate the applicability of our results. 
Example 12. Consider the boundary value problem

$$
\begin{gathered}
-\Delta^{2} u(t-1)+u(t)=\lambda f(t, u(t)) \quad t \in[1,3] \\
u(0)=u(T+1)=0
\end{gathered}
$$

where

$$
\begin{gathered}
f(u)= \begin{cases}\frac{1}{24 \sqrt{2}}, & u \leq \sqrt{2}, \\
97 u^{2 / 3}-97 \cdot(\sqrt{2})^{2 / 3}+\frac{1}{96}, & u>\sqrt{2},\end{cases} \\
F(u)= \begin{cases}\frac{1}{24 \sqrt{2}} u, & u \leq \sqrt{2}, \\
97 \cdot \frac{3}{5} u^{5 / 3}-97 \cdot(\sqrt{2})^{2 / 3} u+\frac{1}{96} u, & u>\sqrt{2} .\end{cases}
\end{gathered}
$$

It is clear that $p=2, q=2, T=3$, and $c=2$. Let $\alpha=\sqrt{2}$, $\beta=4$; it follows that $\max _{u \in[-\sqrt{2}, \sqrt{2}]} F(u)=1 / 24, F(\beta)=$ $F(4)=97 \cdot(4 / 5) \cdot 2^{1 / 3}+(1 / 24)$. Then it can be easily shown that all conditions in Theorem 9 are satisfied. Direct calculations give $\widetilde{\varphi}_{1}=1 / 2$ and $\widetilde{\varphi}_{2}=\left(\left(388 \times 2^{1 / 3}\right) / 400\right) \approx 1.222$, so $\widetilde{\varphi}_{1}<\widetilde{\varphi}_{2}$ and $\Lambda_{3}=\left(1 / \widetilde{\varphi}_{2}, 1 / \widetilde{\varphi}_{1}\right)=((1 / 1.222), 2) \approx(0.8183,2)$. Therefore, (53) has at least three solutions in $E$ provided that $\lambda \in(0.8183,2)$.

\section{Conflict of Interests}

The authors declare that there is no conflict of interests regarding the publication of this paper.

\section{Acknowledgments}

This work was supported by National Natural Science Foundation of China (no. 11101098), the Foundation of Guangzhou Education Bureau (no. 2012A019), PCSIR (no. IRT1226), and Natural Science Foundation of Guangdong Province (no. S2013010014460).

\section{References}

[1] R. P. Agarwal, Difference Equations and Inequalities: Theory, Methods and Applications, Marcel Dekker, New York, NY, USA, 1992.

[2] S. N. Elaydi, An Introduction to Difference Equations, Springer, New York, NY, USA, 1996.

[3] V. Lakshmikantham and D. Trigiante, Theory of Difference Equations: Numerical Methods and Applications, Academic Press, New York, NY, USA, 1988.

[4] R. E. Mickens, Difference Equations: Theory and Application, Van Nostrand Reinhold, New York, NY, USA, 1990.

[5] A. N. Sharkovsky, Yu. L. Maĭstrenko, and E. Yu. Romanenko, Difference Equations and Their Applications, Kluwer Academic Publishers, Dordrecht, The Netherlands, 1993.

[6] W. G. Kelley and A. C. Peterson, Difference Equations: An Introduction with Applications, Academic Press, New York, NY, USA, 1991.
[7] A. Cabada, A. Iannizzotto, and S. Tersian, "Multiple solutions for discrete boundary value problems," Journal of Mathematical Analysis and Applications, vol. 356, no. 2, pp. 418-428, 2009.

[8] A. Iannizzotto and S. A. Tersian, "Multiple homoclinic solutions for the discrete $p$-Laplacian via critical point theory," Journal of Mathematical Analysis and Applications, vol. 403, no. 1, pp. 173182, 2013.

[9] X. Cai and J. Yu, "Existence of periodic solutions for a $2 n$ thorder nonlinear difference equation," Journal of Mathematical Analysis and Applications, vol. 329, no. 2, pp. 870-878, 2007.

[10] Z. Guo and J. Yu, "Periodic and subharmonic solutions for superquadratic discrete Hamiltonian systems," Nonlinear Analysis: Theory, Methods \& Applications A: Theory and Methods, vol. 55, no. 7-8, pp. 969-983, 2003.

[11] X. Deng, X. Liu, Y. Zhang, and H. Shi, "Periodic and subharmonic solutions for a $2 n$ th-order difference equation involving p-Laplacian," Indagationes Mathematicae, vol. 24, no. 3, pp. 613625, 2013.

[12] G. Bonanno, "A critical points theorem and nonlinear differential problems," Journal of Global Optimization, vol. 28, no. 3-4, pp. 249-258, 2004. 


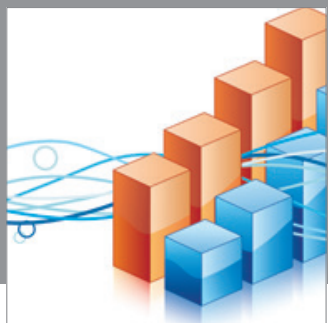

Advances in

Operations Research

mansans

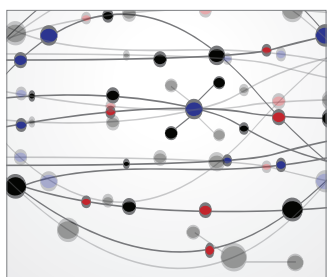

The Scientific World Journal
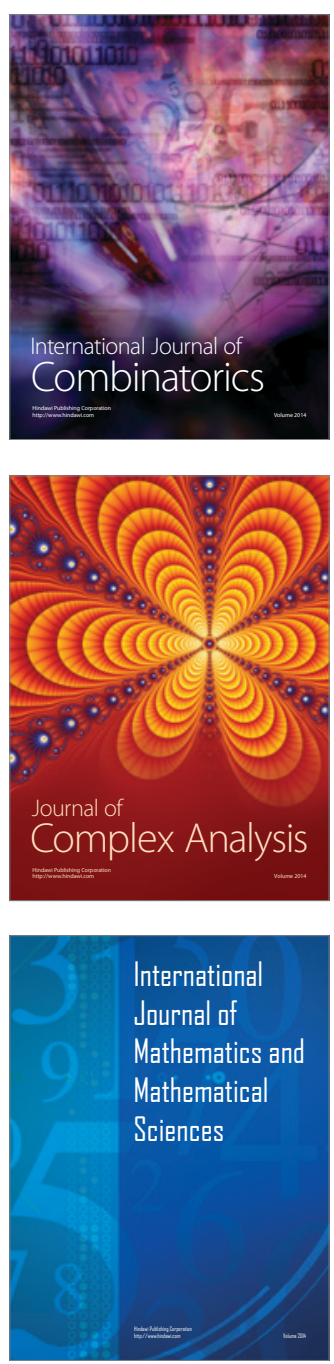
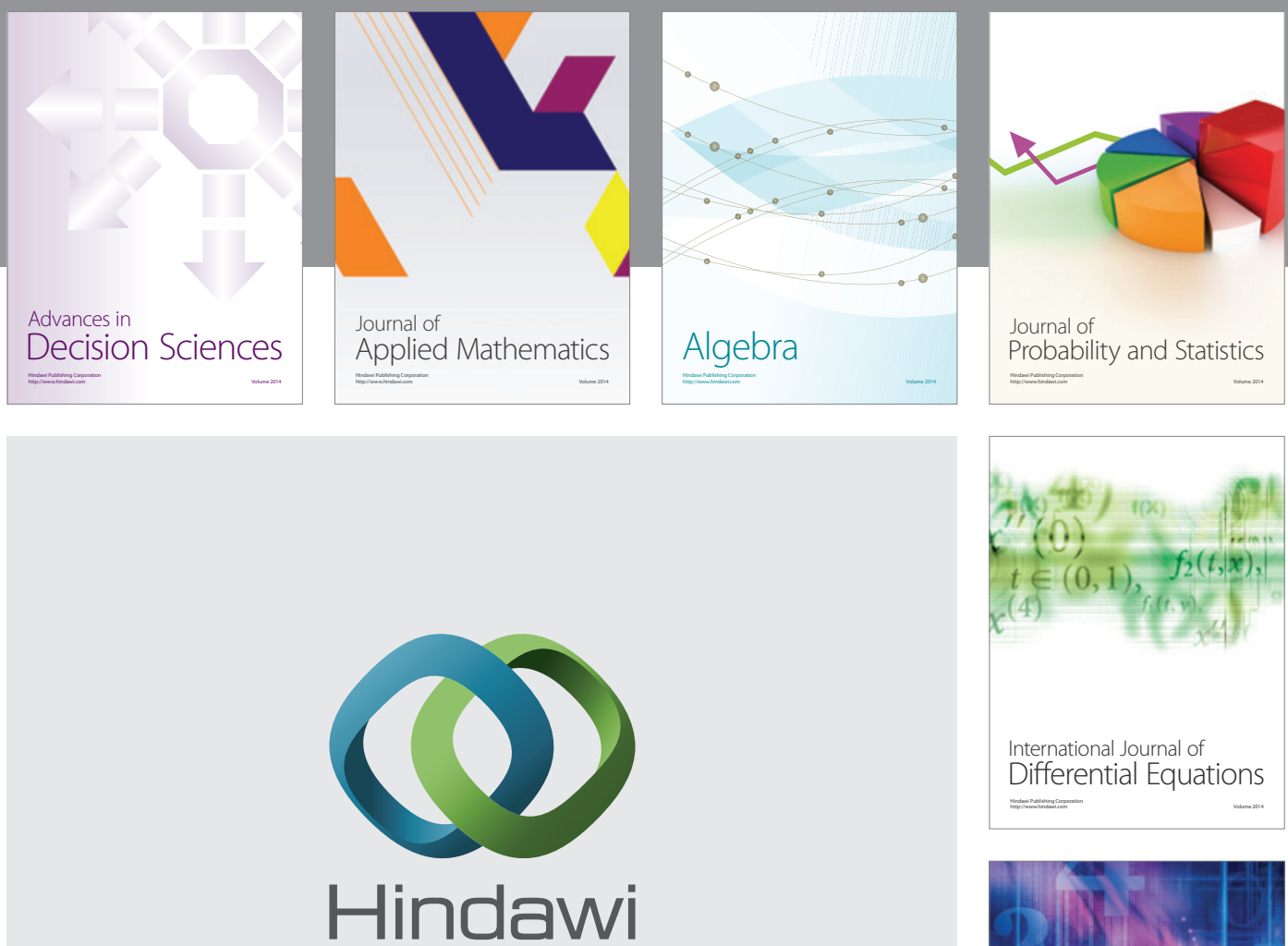

Submit your manuscripts at http://www.hindawi.com
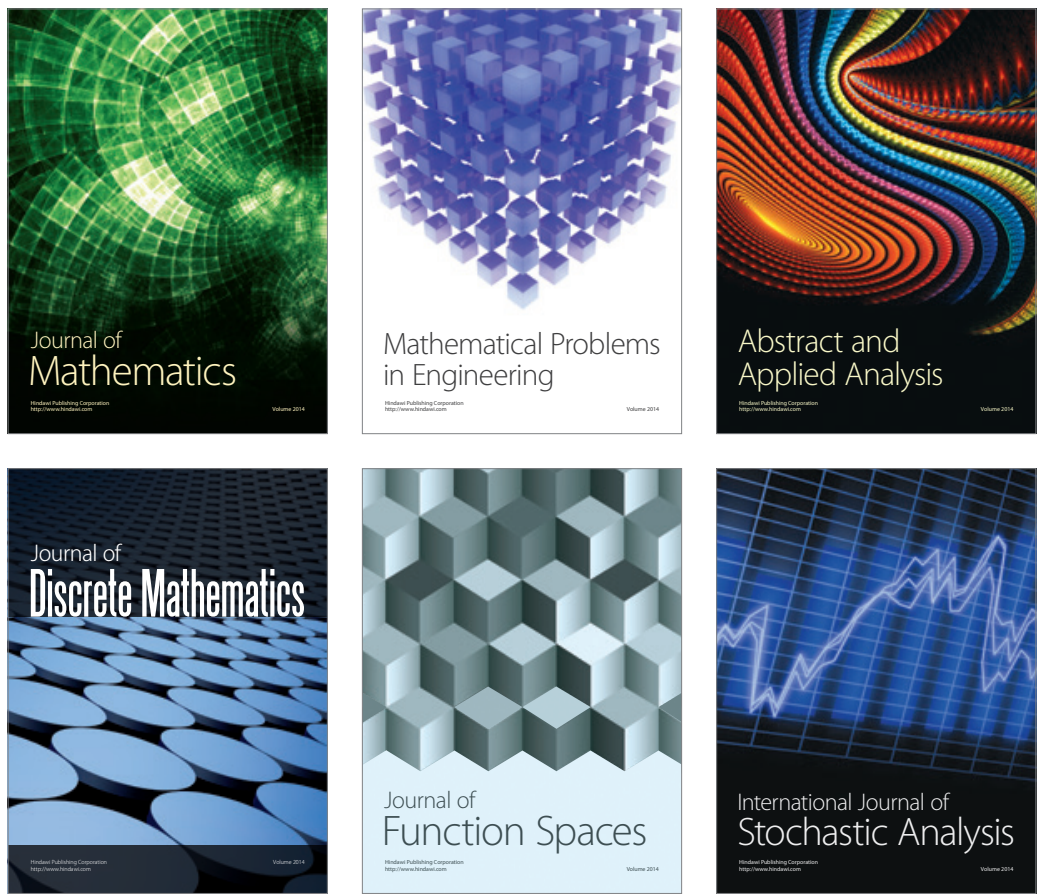

Journal of

Function Spaces

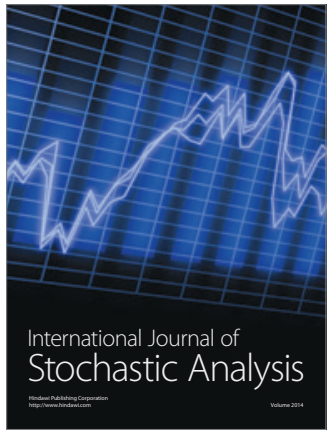

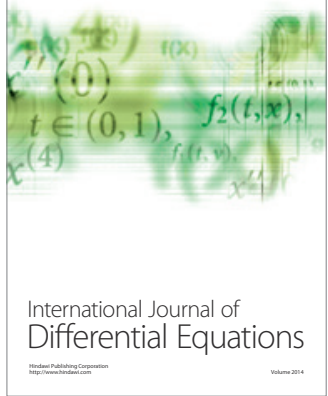
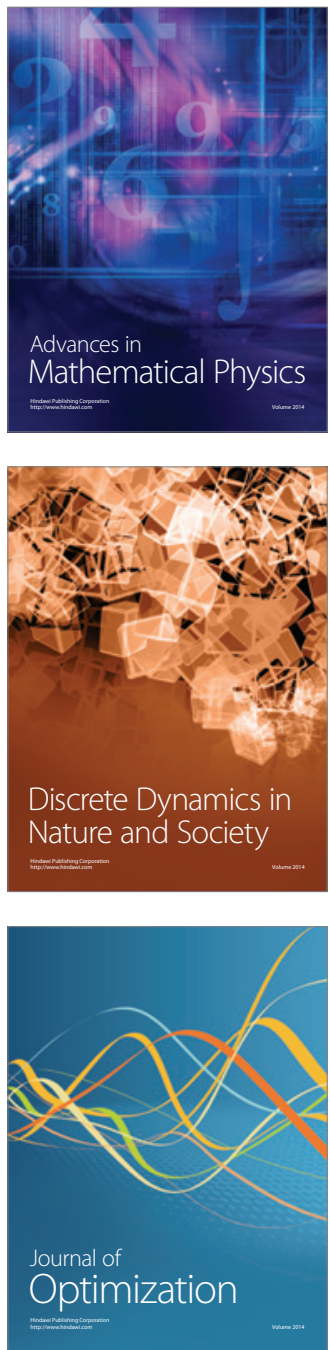